\title{
Wearable IoT based Distributed Framework for Ubiquitous Computing
}

\author{
Dr. V. Suma \\ Professor, \\ Department of Information Science \& Engineering, \\ Dayananda Sagar College of Engineering, \\ Bangalore, India.
}

\begin{abstract}
In Internet of Things (IoT) based systems, the multi-level user requirements are satisfied by the integration of communication technology with distributed homogeneous networks termed as the ubiquitous computing systems (UCS). The PCS demands openness in heterogeneity support, management levels and communication for distributed users. However, providing these features is still a major challenge. In wearable IoT (WIoT) based medical sensors based applications, the end users reliability of communication is enhanced using a scalable distributed computational framework introduced in this paper. The demand and sharing parameters forms the basis of analysis of resource allocation by means of recurrent learning in this framework. The rate of communication may be improved while reducing the time delay for the end users of WIoT based medical sensors with the help of UCS and estimated resource requirements. Other than data transfer, sharing and resource allocation, end-user mobility management may also be performed on the WIoT medical sensors using the proposed framework. Certain metrics are used for proving the consistency of the framework that are assessed with the help of experimental analysis and performance estimation. Parameters inclusive of storage utilization, bandwidth, request backlogs, requests handled, request failure and response time are estimated. Reduced response time, backlogs and request failure with improved storage utilization, bandwidth and requests handled are evident using the proposed framework when compared to the existing models.
\end{abstract}

Keywords: Storage management, scalable network, wearable IoT, ubiquitous computing, medical sensors 
Journal of Ubiquitous Computing and Communication Technologies (UCCT) (2021)

Vol.03/ No.01

Pages: 23-32

https://www.irojournals.com/jucct/

DOI: https://doi.org/10.36548/jucct.2021.1.003

\section{Introduction}

The physical world is transformed into a ubiquitous computing environment due to the deployment and application of ubiquitous systems [1]. IoT system services are developed by integrating the physical world with decision making and computational abilities by means of smart sensing units and wireless devices. Multimedia, communication networks, information access, business, healthcare and other applications for residential and commercial consumers are benefited by the services of this environment [2]. Third party services and applications, communication technologies, distributed and local resources integrated with pervasive computing environment as well as querying requests are improved by providing instant responses and satisfying the requirements of the users. Cloud and other network services are fetched using a layered approach in this environment to ensure the IoT assisted wearable sensors have sufficient availability of resources at the user network edge [3]. User equipment are used for establishing communication with independent and ubiquitous access to resources by the users. Adaptable communication technology is used for connecting the external services and networks to the users by means of the diverse applications available in the user equipment [4-6].

In ubiquitous computing environment, service reliability is expected by the users. Hence, service concentration for pervasive users is an essential requirement [7]. Services are used for providing access to heterogeneous resources in the pervasive computing systems. The communication interfaces of the underlying networks of various service systems are used for accessing the services. The user demands are satisfied by distribution and providing access to ubiquitous computing systems over various computing environment [8]. In the pervasive environment, functions like sharing, resource allocation, query processing, service compositions and concurrent user access are provided. Various IoT based sensor networks are integrated with communication environment with distributed data across various locations and medical sensor data centers [9]. The end users are provided with reliable service by means of including edge computing, distributed servers, medical sensor data centers, mobile and cellular networks and software defined networking (SDN) paradigms. 
Journal of Ubiquitous Computing and Communication Technologies (UCCT) (2021)

Vol.03/ No.01

Pages: 23-32

https://www.irojournals.com/jucct/

DOI: https://doi.org/10.36548/jucct.2021.1.003

\section{Related Works}

The SDN network scalability is enhanced with the help of a service concentrated loadbalancing mechanism [10]. Considering the flow requests, the load across the network is disseminated with the help of the SDN controllers. During load dissemination, the delay constraints are reduced by means of Quality of Service (QoS) routing adopted by the SDN. Minimizing the request backlogs still remains a challenge despite successful leverage of link utilization using this technique. The network service capability is improved using an analytical scalability scheme [5]. The service scalability is analyzed and relationships are modeled by integrating the service evaluation models and network description in a corresponding manner. In case of wearable IoT sensors with heterogeneous communication schemes, architecture and networks with dynamic topology, this technique is efficient [8]. Relationship based resource location and network load handling is performed in an efficient manner using this method.

For application in fifth generation core networks, a resource scaling technique based on machine learning is used [11]. Sensors assist in prediction of data arrival rate in the connected mobile networks thereby improving the traffic forecasting by means of the scaling technique. Resource scalability is improved through traffic dissemination based on arrival rate for construction of neural network training sets [12]. The backlogs caused by traffic forecasting are minimized effectively using this mechanism. In SDN networks, the scalability is improved with the help of open level control (OLC) plane architecture. Under dense traffic conditions, the level of scalability is retained by integrating user domain requirements and heterogeneous service discovery [7]. Under controlled delay, the total service discovery packets count is improved in order to make OLC reliable. The IoT assisted controller and their prolonged decision making is the major drawback in this architecture leading to service response delay [13].

The SDN scalability is improved by a novel hybrid switching (HS) technique. Approximation algorithm is used for achieving performance optimization and scalability for assimilation in HS and conventional SDN [14]. Overhead minimization and network load handling is performed exclusively by the approximation algorithm. The traffic load and flow is controlled for improving the network throughput under lesser number of forwarding 
Journal of Ubiquitous Computing and Communication Technologies (UCCT) (2021)

Vol.03/ No.01

Pages: 23-32

https://www.irojournals.com/jucct/

DOI: https://doi.org/10.36548/jucct.2021.1.003

conditions in the hybrid design. Scalable and flexible resource provisioning is performed in cloud-dependent IoT using medical sensor data transmission assisted by edge-node IoT. For IoT assisted sensors, the resource utilization is improved by accepting incoming traffic through scheduling of the edge node bandwidth in this transmission scheme. User demands are met while processing multiple requests by edge nodes with the help of scheduled bandwidth and replicated cloud resources [15]. Limited requests are handled with less bandwidth utilization through scalability in medical sensor data transmission models.

\section{Proposed Work}

The user and devices with heterogeneous operations are supported by optimizing the intrinsic features like scalability and flexibility of ubiquitous computing systems. The interoperability provided by these systems are of high level. For multiple users, the service support is extended simultaneously by means of a distributed scalable computational framework proposed in this paper. The network communication rates are improved in a precise manner under storage optimization and request processing functions using this framework. The proposed framework and its corresponding network model are analyzed in this paper. Four layers namely cloud, infrastructure, control and ubiquitous are the categories of the proposed framework's network model.

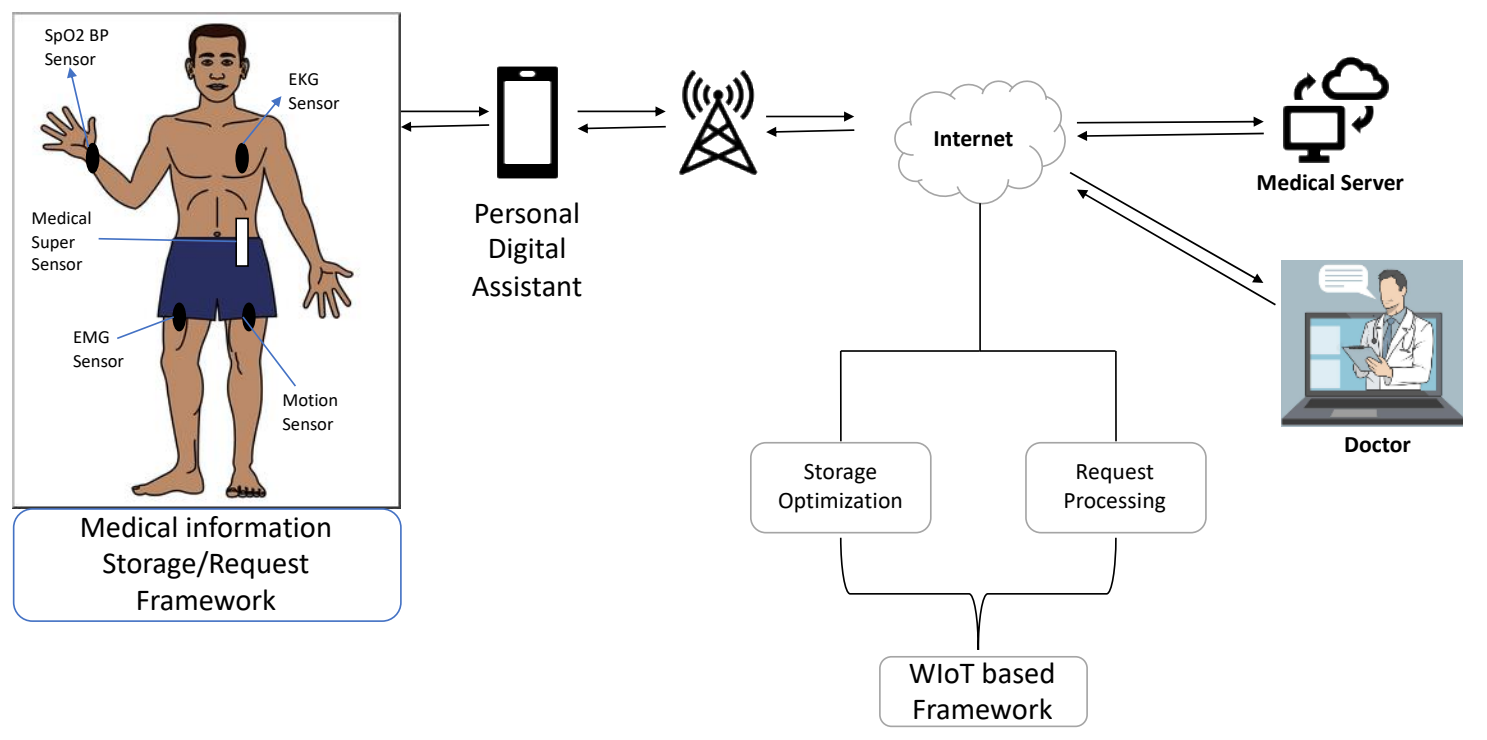

Fig. 1. Proposed WIoT based information processing framework 
Journal of Ubiquitous Computing and Communication Technologies (UCCT) (2021)

Vol.03/ No.01

Pages: 23-32

https://www.irojournals.com/jucct/

DOI: https://doi.org/10.36548/jucct.2021.1.003

Figure 1 provides the architecture of the proposed framework. Storage optimization and request processing are the two major functions of this framework. Despite the restrictions in the availability of resources, the queries are delivered to the cloud layer through the request processing function. Bluetooth or Zigbee platform is used to execute this function with the help of personal device assistance (PDA). The sensors have limited resource availability and insufficient request handlers. However, the delivery and request processing are performed in a loss-less and punctual manner. From the ubiquitous environment, seamless flow is ensured while minimizing the request blocking probability. Through internet, the sensor data is transferred to the medical server at the cloud environment of the infrastructure layer in the ubiquitous environment which in turn operates in the control layer of the framework. The request flow is improved while minimizing the request transmission bottlenecks in the control layer by performing initial request processing. In resource allocation, backlogs and failures are prevented by storage allocation based on density variation at the infrastructure layer by means of storage optimization.

\section{Results and Discussion}

Around 500 IoT assisted wearable medical sensor based devices are used for analysis of the performance of the proposed model in a logic scenario using Riverbed modeler. Files may be retrieved from open cloud based on the request from devices using file sharing application. The configurations and functional parameters are analyzed in a detailed manner. Metrics inclusive of storage utilization, bandwidth, request backlogs, density of requests, request failure and response rate are compared for comparative performance analysis. Cloudlet, Fog computing, mobile edge computing and transparent computing IoT based paradigms are compared with the proposed model to verify its reliability. Retransmission requires lesser time due to large number of resource allocation requests delivered at the cloud. The sum of service time and processing time should be less than the maximum time for mapping the appropriate resources with the requests. The difference between total requests and change in requests are pipelined by storage optimization while selecting infrastructure units with less utilized storage of the units. 


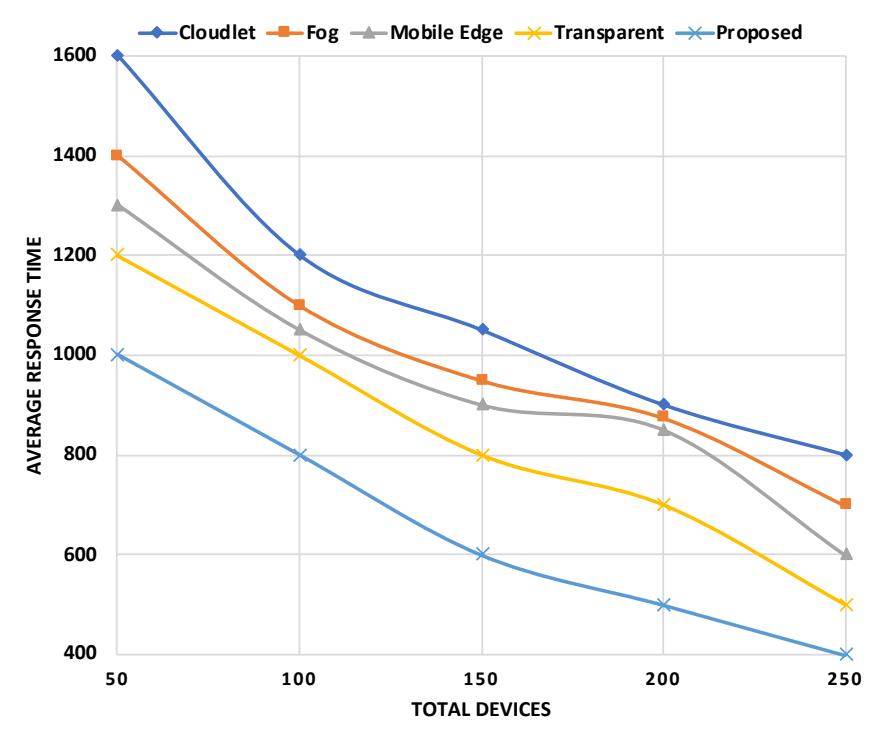

Fig. 2. Response time comparison of various devices

Fewer requests are reinitiated in the proposed model due to the aforementioned reasons. Depending on the incoming request order, spontaneous resource allocation is performed in the cloud layer. The internal bottleneck issues are minimized by streamlining the requests in the pervasive environment control layer. When compared to other SDN controllers, the processing time is lesser for the proposed model. Lesser processing time variations are identified while maintaining the incoming and propagation requests with the help of the request balancing factor. The delivery requests and their service rate is improved by allocation of appropriate storage thereby balancing the requests for multiple devices. The response time is minimized and the requests are served on time using the proposed model. Figure 2 provides the response time comparison with respect to various devices.

Figure 3 provides the comparison of the existing systems and proposed framework with respect to the request backlogs. The communication backlogs increases on processing and the resources are not mapped with the total requests in the conventional systems. Within the maximum time, instantaneous resource allocation is performed to the delivered requests using the proposed framework. At the cloud layer, a delay is evident during delivery or retransmission of the requests. These requests have to wait for a longer duration and are not mapped with the resources. Occurrence of backlog and drop in the request are seen when the 
response time is lesser than the maximum time. The product of total requests and devices and rate of arrival decides the organization of incoming and outgoing requests in the proposed model. High resource allocation and successful request delivery count is ensured by maintaining the request delivery in the transmission process with precise detection. This enables processing all requests within the specified time while minimizing the backlogs with the proposed framework.

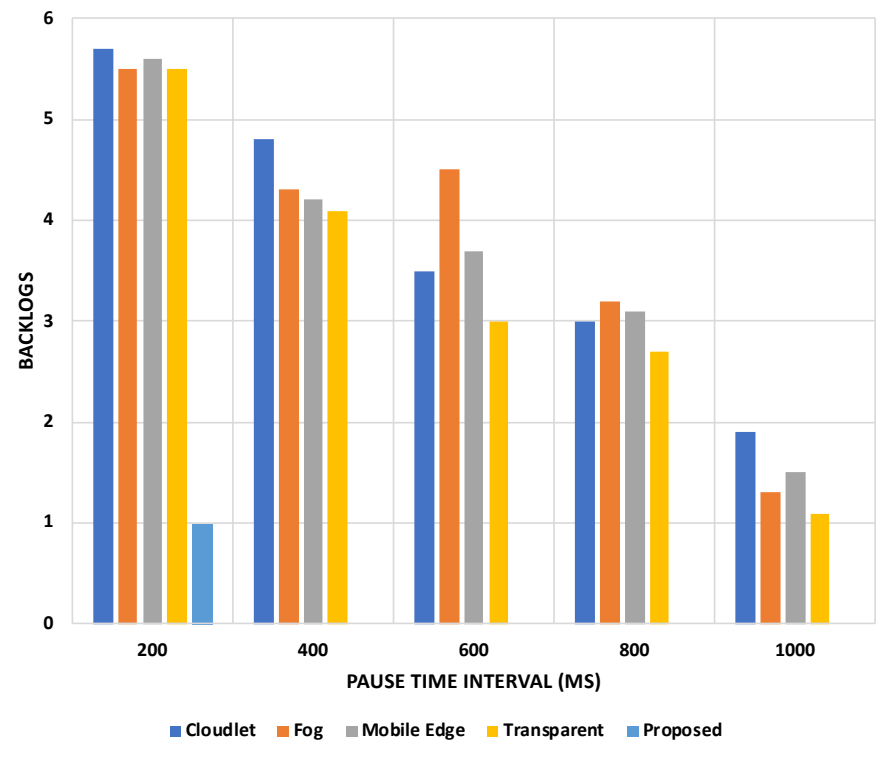

Fig. 3. Comparison based on request backlogs

Higher bandwidth utilization is achieved by increasing the number of requests handled and request response time. High external bandwidth is allocated for the requests due to the handling of large number of requests at the control plane in the proposed framework. Stagnant-less propagation is ensured by the balancing factor at the control plane after processing and seamlessly discharging the requests. Based on the change in requests, storage is assigned and the request delivery is improved at the cloud layer by storage optimization. In successive flows, higher requests are facilitated by resource allocation while ensuring minimum backlogs due to pipelining of requests. Maximum utilization of the bandwidth allocated between the infrastructure and control layer is ensured for handling the increased flow of requests. The maximum link available for communication is greater than 1 leading to bandwidth utilization increase. The incoming requests are handled by increasing the total links available. Figure 4 
Journal of Ubiquitous Computing and Communication Technologies (UCCT) (2021)

Vol.03/ No.01

Pages: 23-32

https://www.irojournals.com/jucct/

DOI: https://doi.org/10.36548/jucct.2021.1.003

represents the comparison of maximum response time and bandwidth utilization of various models.

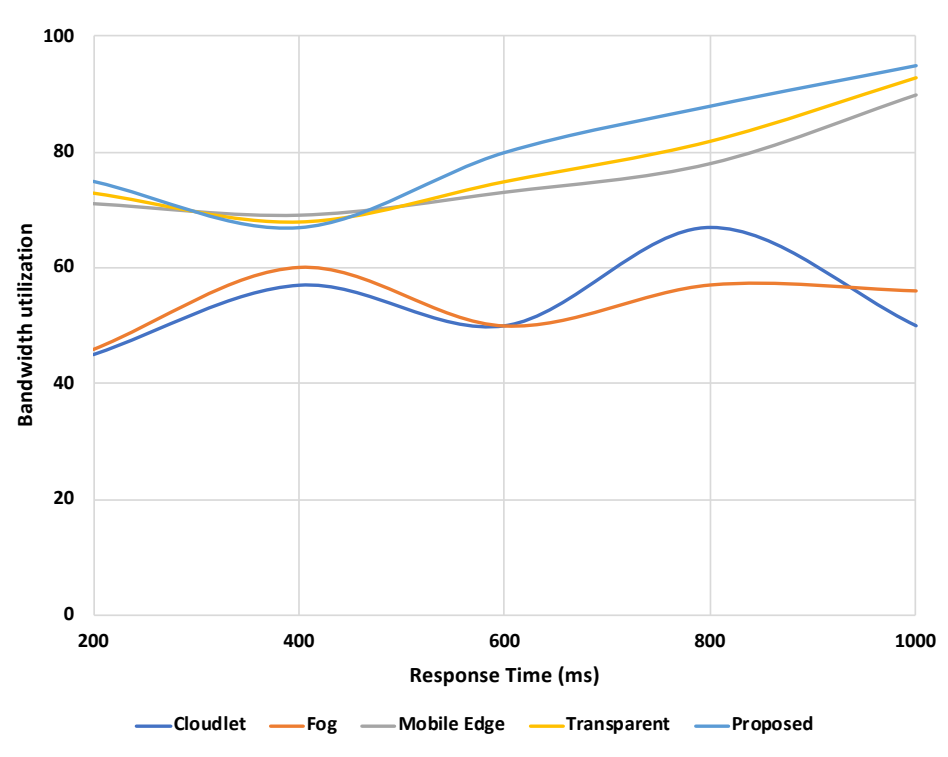

Fig. 4. Comparison of Bandwidth Utilization

Based on the storage availability, the processed requests are forwarded from the control layer to the infrastructure layer. The network storage utilization is increased with the increase in response time. Unnecessary cloud layer request loss is prevented by this measure. The conditions for transmission is satisfied by categorization of storage requirements. If the number of requests is greater than the storage size, pipelining of requests to the next storage infrastructure is carried out. Request drops and overloading is prevented by selection of successive infrastructure storage by utilization rate analysis. The constraints and capacity of available storages are analyzed for storing successive requests. The storage utilization is increased when there is a large number of request flow for maximum bandwidth utilization. High resource allocation rate is caused by the maximum level storage utilization.

\section{Conclusion}

In wearable medical sensors with IoT assistance, the reliability of communication of ubiquitous systems are improved by the proposed scalable and distributed computational framework proposed in this paper. Reliability is maintained in request handling at the pervasive 
Journal of Ubiquitous Computing and Communication Technologies (UCCT) (2021)

Vol.03/ No.01

Pages: 23-32

https://www.irojournals.com/jucct/

DOI: https://doi.org/10.36548/jucct.2021.1.003

environment and its control layer by implementing the distributed framework. Communication error is detected and minimized using recurrent learning process by the framework. Requests are disseminated in an uninterrupted manner by controlling the infrastructure layer factors, namely storage allocation and request processing by the framework. Maximum request processing and bandwidth utilization is retained by modelling the request flow density and support varying device through the framework's learning process. Under controlled response time, the storage utilization is improved while backlogs and request failures are minimized according to the experimental results proving the consistency of the proposed framework. Future work is directed towards extension of the scalable framework to edge computing network. Concurrent heterogeneous applications may be supported by further improving the scalability and flexibility of the framework.

\section{References}

[1] Cena, F., Likavec, S., \& Rapp, A. (2019). Real world user model: Evolution of user modeling triggered by advances in wearable and ubiquitous computing. Information Systems Frontiers, 21(5), 1085-1110.

[2] Chen, Y., Qin, X., Wang, J., Yu, C., \& Gao, W. (2020). Fedhealth: A federated transfer learning framework for wearable healthcare. IEEE Intelligent Systems, 35(4), 83-93.

[3] Wan, J., Al-awlaqi, M. A., Li, M., O’Grady, M., Gu, X., Wang, J., \& Cao, N. (2018). Wearable IoT enabled real-time health monitoring system. EURASIP Journal on Wireless Communications and Networking, 2018(1), 1-10.

[4] Kong, X., Tong, S., Gao, H., Shen, G., Wang, K., Collotta, M., ... \& Das, S. (2020). Mobile edge cooperation optimization for wearable internet of things: a network representationbased framework. IEEE Transactions on Industrial Informatics.

[5] Li, S., Zhang, B., Fei, P., Shakeel, P. M., \& Samuel, R. D. J. (2020). Computational efficient wearable sensor network health monitoring system for sports athletics using IoT. Aggression and Violent Behavior, 101541.

[6] Khowaja, S. A., Prabono, A. G., Setiawan, F., Yahya, B. N., \& Lee, S. L. (2018). Contextual activity based Healthcare Internet of Things, Services, and People (HIoTSP): An architectural framework for healthcare monitoring using wearable sensors. Computer Networks, 145, 190-206. 
Journal of Ubiquitous Computing and Communication Technologies (UCCT) (2021)

Vol.03/ No.01

Pages: 23-32

https://www.irojournals.com/jucct/

DOI: https://doi.org/10.36548/jucct.2021.1.003

[7] Friday, N. H., Al-garadi, M. A., Mujtaba, G., Alo, U. R., \& Waqas, A. (2018, March). Deep learning fusion conceptual frameworks for complex human activity recognition using mobile and wearable sensors. In 2018 International Conference on Computing, Mathematics and Engineering Technologies (iCoMET) (pp. 1-7). IEEE.

[8] Janeera D.A., Sasipriya S. (2021) A Brain Computer Interface Based Patient Observation and Indoor Locating System with Capsule Network Algorithm. In: Chen JZ., Tavares J., Shakya S., Iliyasu A. (eds) Image Processing and Capsule Networks. ICIPCN 2020. Advances in Intelligent Systems and Computing, vol 1200. Springer, Cham.

[9] Baig, M. M., Afifi, S., GholamHosseini, H., \& Mirza, F. (2019). A systematic review of wearable sensors and IoT-based monitoring applications for older adults-a focus on ageing population and independent living. Journal of medical systems, 43(8), 1-11.

[10] Bhatt, S., Patwa, F., \& Sandhu, R. (2017, October). An access control framework for cloud-enabled wearable internet of things. In 2017 IEEE 3rd International Conference on Collaboration and Internet Computing (CIC) (pp. 328-338). IEEE.

[11] Bayo-Monton, J. L., Martinez-Millana, A., Han, W., Fernandez-Llatas, C., Sun, Y., \& Traver, V. (2018). Wearable sensors integrated with Internet of Things for advancing eHealth care. Sensors, 18(6), 1851.

[12] Manoharan, S. REVIEW ON UBIQUITOUS CLOUDS AND PERSONAL MOBILE NETWORKS.

[13] Smys, S., \& Ranganathan, G. (2020). Performance Evaluation of Game Theory Based Efficient Task Scheduling For Edge Computing. Journal of ISMAC, 2(01), 50-61.

[14] Smys, S. (2020). A Survey on Internet of Things (IoT) based Smart Systems. Journal of ISMAC, 2(04), 181-189.

[15] Anand, J. V. (2019). Design and development of secure and sustainable software defined networks. Journal of Ubiquitous Computing and Communication Technologies (UCCT), $1(02), 110-120$. 